\title{
Soll der Arzt während der Abteilungsvisite stehen oder sich ans Krankenbett setzen?
}

Rolf H. Adler

Korrespondenz:

Prof. em. Dr. med. Rolf H. Adler

Leiserenweg 4

CH-3122 Kehrsatz

rolf.adler@tele2.ch
In einer Abteilung für Innere Medizin habe ich mir auf der Chefvisite ans Bett eines jeden Kranken einen Stuhl reichen lassen und mich hingesetzt, unabhängig von der verfügbaren Zeit. Für die anschliessende körperliche Untersuchung bin ich selbstverständlich aufgestanden. An einem Klinikabend haben mir meine Mitarbeiter wohl in diesem Zusammenhang einen Melkstuhl geschenkt.

Während ich diesen Artikel entwerfe, sind 10 Tage vergangen seit dem Einsetzen einer Zweitprothese in meine linke Hüfte, nachdem die erste mir $61 / 2$ Jahre lang ein einwandfreies Leben mit leichten sportlichen Aktivitäten ermöglicht hatte. Acht Tage vor der jetzigen Operation hatten zunehmende Schmerzen begonnen. Die Diagnose einen Tag vor der Operation lautete: totaler Querbruch des Prothesenschafts. Der Eingriff dauerte 31/2 Stunden, mehr als eine Stunde habe allein die Entfernung des distalen Fragments von proximal her gedauert. Das präoperative $\mathrm{Hb}$ von $16 \%$ fiel postoperativ auf 8,5\%. Ein grosses Hämatom im Oberschenkel war damit verbunden. Die postoperative Betreuung war ausgezeichnet, lediglich auf eine Einzelheit des Verhaltens des Abteilungsarztes möchte ich eingehen, weil ich eine Änderung im Visitenverhalten der Ärzte vorschlagen möchte.

In den ersten Stunden und Tagen erlebte ich den am Bett stehenden Arzt weit weg und weit oberhalb von mir, wie ich passiv, unbeweglich, mit dem linken Bein in einer Schiene, an einer Infusion und mit Drain im Oberschenkel weit unten wie in einer Gruft lag. Ich fühlte mich verlassen, hilflos und unfähig, auf den Ablauf der Visite Einfluss zu nehmen. Nach der Palpation des Oberschenkels, der Verordnung von Blutbild und Kontrollröntgen zog der Arzt mit seinem Unterassistenten weiter. Durch sein Stehen an meinem Bett hatte ich fortwährend das Gefühl, er sei pressiert, mit den Gedanken schon bei einer nächsten Tätigkeit. Meine Fragen (als Internist) nach dem durchschnittlichen Zeitverlauf eines grossen Hämatoms, etwaigen Residuen, z. B. in Form von Verkalkungen, der zeitlichen Erholung des Hb-Spiegels, dem Schicksal einer Zweitprothese bezüglich Lockerung blieben meinerseits, eingeschüchtert, ungeäussert.
Hätte er sich hingesetzt, wäre unsere Beziehung weniger «schief» gewesen, hätte «Zeit zur Verfügung haben» bedeutet, hätte mich zum Fragenstellen angeregt und mir das Gefühl vermittelt, noch ein Mensch zu sein im Kreis von andern Menschen und weniger nur der anonyme Besitzer eines für den Arzt bedeutsamen Körperteils.

Um die Ängste und Bedürfnisse eines Patienten zu fühlen und zu verstehen, helfen dem Arzt eigene Erfahrungen als Patient und die Einfühlung in den Patienten, also die Empathie. Sie bringen die Erkenntnis, dass der Patient, je beeinträchtigter und je schwerer krank oder behindert er ist, umso mehr in ein Denken und Fühlen verfällt, das früheren Entwicklungsstadien entspricht, wo Hilflosigkeit, Angst vor dem Alleinsein, fehlende Kompetenz und Kontrolle sowie Liegen typisch waren. Mit andern Worten, er verfällt der Regression (Begriff aus der psychoanalytischen Theorie). Der Arzt, der sich ans Krankenbett setzt, fördert die Rückkehr aus der Regression, weil er den Kranken als gleichberechtigten Partner annimmt. Den Teil der Regression, den auch er im Moment nicht aufheben kann, erfordert von ihm die Ergänzung der fehlenden Möglichkeiten des Patienten. Arzt und Patient bilden eine Einheit, die Einheit des Überlebens. Sie ist besonders klar zu erkennen, wenn er die vitalen Funktionen bereitstellt, die der Kranke nicht erbringen kann.

Die Einheit des Überlebens lässt sich an der Mutter-Säuglings-Beziehung besonders deutlich erkennen. So sagte D. Winnicott: «Es gibt nicht hier eine Mutter und dort ein Kind. Es gibt nur die Einheit des Überlebens.» [1] Je inkompetenter der Säugling, desto mehr muss die Mutter bieten, und wenn der Säugling und später das Kleinkind kompetenter werden, darf die Mutter sich zunehmend der Unterstützung und Ergänzung enthalten.

Auf die Arzt-Patienten-Beziehung zurückübersetzt: Durch das Sichhinsetzen ans Krankenbett verhilft der Arzt dem Kranken zu einer mindestens teilweisen Rückkehr aus der Regression zur Kompetenz, soweit dies der Zustand des Patienten erlaubt. Im Sitzen vermag er zudem das tiefe, zeitlebens vorhandene Bedürfnis nach Bin- 
dung eines Menschen, das J. Bowlby so klar herausgearbeitet hat, besser zu stillen als im Stehen [2]. Er passt sich damit in die Überlebenseinheit Patient-Arzt ein.

Als Arzt muss er sich seines empathischen Verhaltens und Zulassens eines Gesprächs zwischen zwei Partnern übrigens nicht schämen. Hat doch Martin Buber auf die Frage, welches
Gespräch das wichtigste sei, das er in seinem Leben geführt habe, geantwortet: Das Gespräch, in dem ich mich eben befinde.

\section{Literatur}

1 Winnicott DW. Reifungsprozesse und fördernde Umwelt. München: Kindler; 1974.

2 Bowlby J. Bindung. München: Kindler; 1975.

\section{Hirnforschung und Drogenpolitik}

\section{Benjamin Fässler}

\section{Geist - Gesellschaft - Droge}

Über das einseitige und oberflächliche Denken Solothurn: Nachtschatten-Verlag 284 Seiten

Fr. 38.-

ISBN 978-3-0378-8138-5

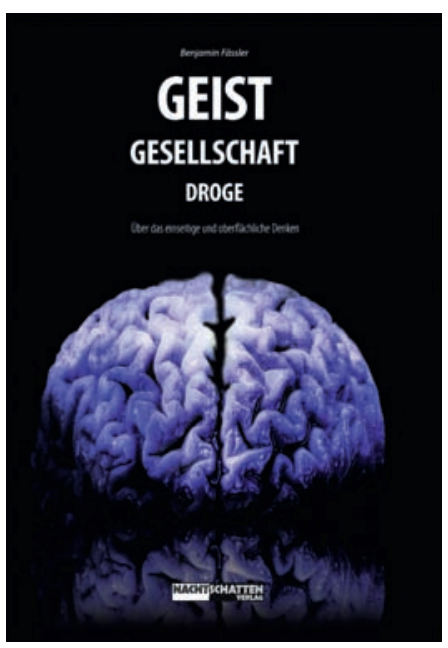

Der Kollege B. Fässler schrieb 1997 ein erstes (inzwischen vergriffenes) Buch «Drogen zwischen Herrschaft und Herrlichkeit». Nun hat der Autor den Inhalt dieses Werkes durch weitere Themen in eine Gesamtschau integriert.
Benjamin Fässler hinterfragt unser Denken und gelangt zur Kernthese, dass die grossen Probleme unserer Zivilisation auf einem erkenntnistheoretischen Grundproblem beruhen, nämlich auf dem einseitigen und oberflächlichen Denken. Einseitig ist das Denken, weil das Hauptgewicht auf das Verstandesmässige gelegt und die Elemente der Intuition und der Gefühle stark verdrängt werden. Oberflächlich ist das Denken, weil es oft unkontrolliert erfolgt, d.h. nicht genügend hinterfragt wird. Um diese These zu untermauern, werden zunächst Wesen, Funktionsweise und Grenzen der drei Wahrnehmungsfunktionen nach C. G. Jung, Denken, Fühlen und Intuition, in einer umfassenden Analyse besprochen.

Erkenntnisse aus der Hirnforschung beleuchten die dargestellten Aspekte aus naturwissenschaftlicher Sicht und verhelfen dazu, sie verständlicher zu machen.

Ein Kapitel beschäftigt sich mit der Frage der Wahrheit. Es wird aufgezeigt, dass die menschliche Erkenntnisfähigkeit ihre Grenzen hat. Daraus folgt, dass der Mensch nie zu einer absoluten Wahrheit, jedoch zu verschiedenen brauchbaren relativen Wahrheiten gelangen kann.

Aus der Kultur- und Geistesgeschichte der Menschheit wird ersichtlich, wie das Verstandesmässige im Laufe der Zeit immer mehr Oberhand gewann. Dies wird am Beispiel der Entwicklung der Religion vom Schamanismus zum Christentum beleuchtet.

Schliesslich wird auf das Thema «Drogen» und «Drogenpolitik» eingegangen. Das Drogen- thema zeigt wohl wie kein anderes das einseitige und oberflächliche Denken auf - die herrschenden Ansichten bewegen sich oft im Bereich zwischen Halbwahrheiten und Ganzfalschheiten -, und dies hat zur verfehlten Drogenpolitik geführt. Durch eine sinnvolle Liberalisierung werden bestimmt nicht alle Drogenprobleme aus der Welt geschafft, unter bestimmten Voraussetzungen könnte sie aber einen Ausweg aus dem heutigen Dilemma aufzeigen.

B. Fässler hat als Kardiologe für einmal keine individuelle Herzrhythmusstörung diagnostiziert, sondern den Puls des globalen Kreislaufs gefühlt. Wir sind zu einer Sucht-, Konsum-, Überfluss- und Leistungsgesellschaft verkommen und einem Machbarkeitswahn verfallen. Die gegenwärtige globale Finanz- und Wirtschaftskrise resultiert aus einem rein materialistisch ausgerichteten Erfolgsdenken, in dem die Jung'schen Funktionen Gefühl und Intuition zu kurz kommen.

Nach eingehendem diagnostischem Bemühen von Dr. Fässler (550 Quellenangaben!) bleibt zu hoffen, dass auch Therapien eingeleitet werden. Dem Autor dieses anspruchsvollen Buches wäre zu wünschen, dass er vom Rufer in der Wüste zum Animator für umfassende und wirksame Veränderungen unseres Umfeldes mutiert.

Max Schreier, Kriegstetten 